03

\title{
Определение распределения интегральной плотности при маховском отражении ударных волн
}

\author{
(C) А.М. Шевченко ${ }^{1}$, Ал.А. Павлов ${ }^{1}$, А.А. Павлов ${ }^{2}$, М.П. Голубев ${ }^{1}$, \\ Д.В. Хотяновский ${ }^{1}$, А.С. Шмаков ${ }^{1}$ \\ ${ }^{1}$ Институт теоретической и прикладной механики им. С.А. Христиановича \\ СО РАН, Новосибирск \\ ${ }^{2}$ Новосибирский государственный университет \\ E-mail: shevch@itam.nsc.ru
}

Поступило в Редакцию 22 декабря 2016 г.

Представлены методика и результаты определения поля интегральной плотности при изучении структуры течения, соответствующего маховскому взаимодействию ударных волн при числе Маха 3. Оптическая диагностика течения осуществлялась интерференционным методом на основе самонаводящихся фильтров Цернике (метод НП АВТ). Численное моделирование выполнено с помощью комплекса программ CFS3D для решения уравнений Эйлера и Навье-Стокса. Впервые получены количественные данные о распределении интегральной по пути зондирующего излучения плотности при одном направлении просвечивания трехмерного течения в области маховского взаимодействия ударных волн.

DOI: $10.21883 /$ PJTF.2017.10.44619.16615

При проведении экспериментов в сверхзвуковых аэродинамических трубах широко применяются оптические методы диагностики. Однако большинство из них используется для визуализации течения, т.е. получения качественной информации о структуре потока в исследуемой области. Например, при изучении взаимодействия ударных волн с образованием тройных конфигураций [1-6] и, в частности, маховского отражения [7-9] выполнено большое число расчетно-теоретических и экспериментальных исследований. При этом в экспериментах, как правило, проводилась только визуализация течения, и все количественные оценки получены для геометрических параметров (углы наклона клиньев, скачков уплотнения и т.п.). 
Однако интерференционные методы позволяют получать не только качественную, но и количественную информацию об изменении длины оптического пути зондирующего излучения, проходящего через исследуемую неоднородность. Изменение плотности воздуха в потоке приводит к смещению интерференционных полос на интерферограмме. Пусть до включения потока разность порядков интерференции (номеров интерференционных полос) для точек $(x, y)$ и $\left(x_{0}, y_{0}\right)$ на регистрируемом изображении составляла $\Delta N_{0}=N(x, y)-N\left(x_{0}, y_{0}\right)$. Тогда после включения потока будем иметь

$$
\begin{aligned}
& \Delta N(x, y)=\Delta N_{0}+\frac{S(x, y)-S\left(x_{0}, y_{0}\right)}{\lambda} \\
& =\Delta N_{0}+\frac{\xi}{\lambda} \int_{0}^{L}\left[\rho(x, y, z)-\rho\left(x_{0}, y_{0}, z\right)\right] d z,
\end{aligned}
$$

где $\lambda$ - длина волны зондирующего излучения; $\xi-$ постоянная Гладсона-Дэйла; $L-$ ширина рабочей части установки; $\rho-$ плотность воздуха; $S$ - длина оптического пути.

Формально путем решения интегрального уравнения (1) можно найти пространственное распределение плотности в исследуемом течении. Однако очевидно, что по одному подобному уравнению получить общее решение невозможно. Для этого необходимо иметь континуум уравнений для направлений просвечивания неоднородности в диапазоне $180^{\circ}$ [10]. И только в некоторых случаях, например для объектов с осевой симметрией, возможно получение решения по данным одного направления просвечивания. При исследовании трехмерных течений в аэродинамических трубах, как правило, весьма затруднительно получить экспериментальные данные даже для нескольких направлений просвечивания.

С другой стороны, в настоящее время существуют эффективные методы численных расчетов для сложных трехмерных потоков. Сравнение результатов расчетов и эксперимента позволило бы судить об эффективности как экспериментальной методики, так и алгоритма расчета. Но указанные выше причины не позволяют экспериментально получить информацию о полях плотности, необходимую для сравнения с численными расчетами. Однако для валидации получаемых результатов нет необходимости решать обратную задачу для нахождения 
распределения полей плотности по интерферометрическим данным. Более правильно проводить прямое сравнение сдвига полос, полученное из численных расчетов, с экспериментальными данными.

Задача численного определения сдвига полос является прямой, и ее решение может быть легко получено из найденного расчетным путем распределения плотности. Это позволяет исключить необходимость решения обратной, условно некорректной задачи, по нахождению распределения плотности используя интегральные уравнения (1). Даже для трехмерных объектов и для одного направления просвечивания совпадение численных и экспериментальных значений $\Delta N$ позволяет более уверенно судить как об адекватности численных расчетов, так и о применимости экспериментальной методики.

В настоящей работе представлен пример комплексного подхода к изучению сложного течения - маховского отражения ударных волн. Эксперименты выполнены в сверхзвуковой аэродинамической трубе Т-325 ИТПМ СО РАН. Для формирования системы ударных волн разной интенсивности использовалась модель из двух встречных клиньев с острой кромкой. Ширина клиньев составляла $100 \mathrm{~mm}$. Отношение размаха клина к хорде 3.25. Представленные в работе экспериментальные данные получены при числе Маха $\mathrm{M}=3$, полном давлении в форкамере $0.21 \mathrm{MPa}$, температуре торможения $282 \mathrm{~K}$. Единичное число Рейнольдса, соответствующее условиям эксперимента, составляло $17 \cdot 10^{6} \mathrm{~m}^{-1}$. Угол отклонения потока на скачке уплотнения составлял $23^{\circ}$.

Регистрация интерферограмм осуществлялась с использованием метода НП АВТ (НП - насыщение поглощения; АВТ — адаптивный визуализирующий транспарант) [11]. Данный метод является развитием метода АВТ, описанного в $[10,11]$. НП АВТ - самонаводящийся фильтр типа Цернике представляет собой тонкий слой (около $50 \mu \mathrm{m}$ ), изготовленный из поглощающего вещества, размещенный в фокальной плоскости приемного коллиматора шлирен-системы вместо ножа Фуко. Фильтр просветляется под действием сфокусированного зондирующего излучения. Эффект просветления является нелинейным и существенен только при достаточно высокой интенсивности излучения.

В нашем случае основная доля излучения, прошедшего через исследуемую неоднородность, приходится на низкие пространственные частоты и фокусируется в точке, соответствующей фокусировке исходного пучка. Именно в этой точке происходит просветление НП АВТ.

Письма в ЖТФ, 2017, том 43, вып. 10 

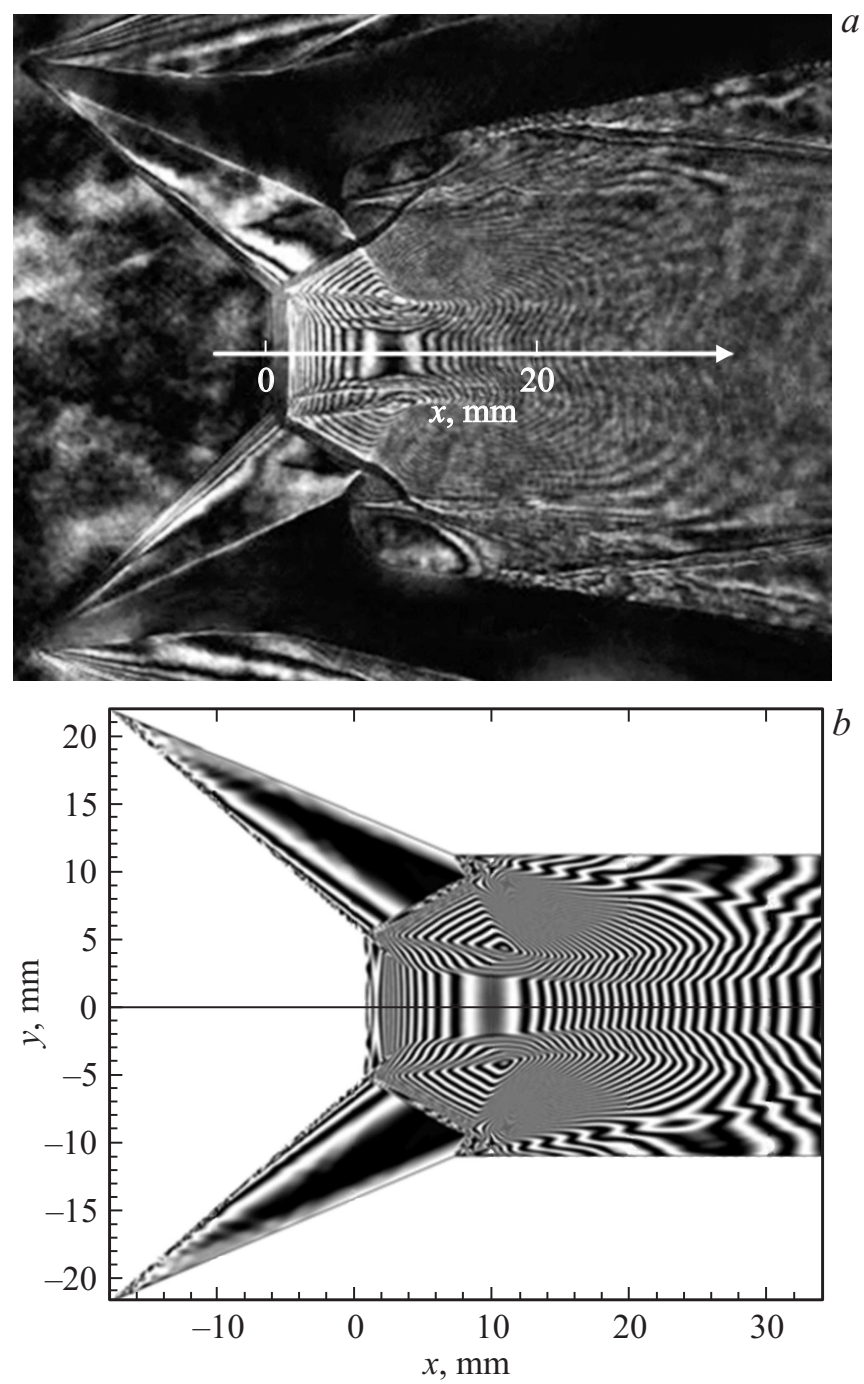

Рис. 1. Интерферограммы течения: $a-$ эксперимент; $b-$ расчет.

Письма в ЖТФ, 2017, том 43, вып. 10 

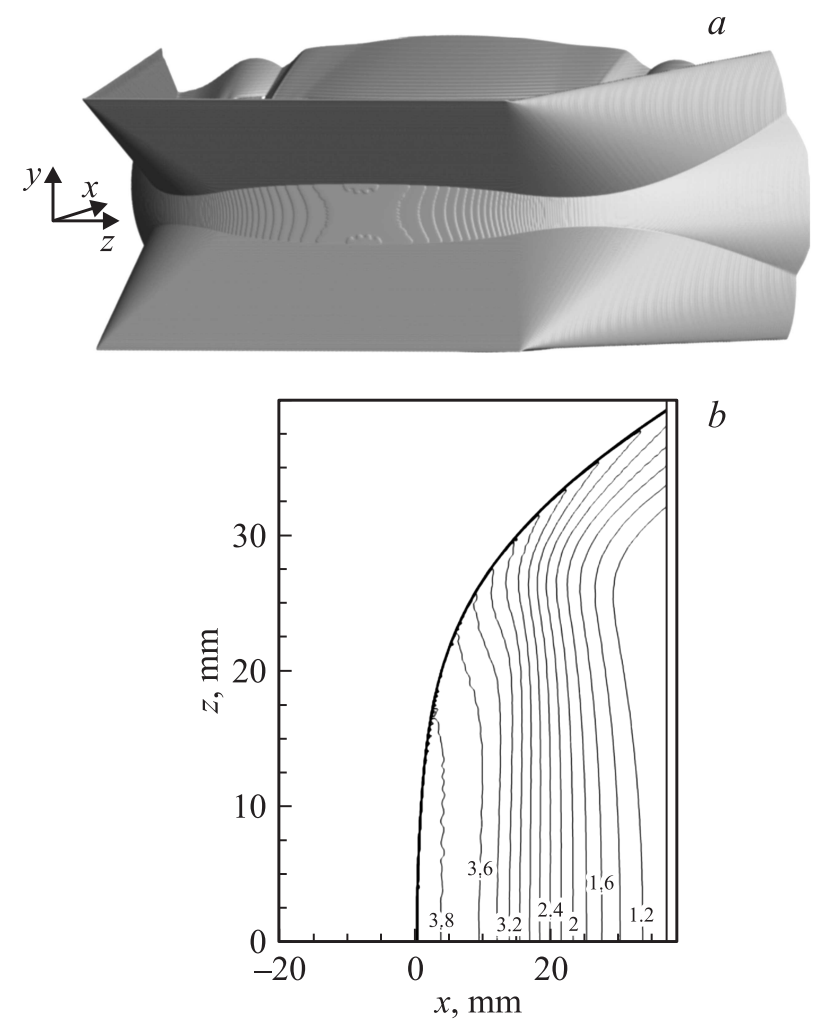

$b$

Рис. 2. Результаты численного моделирования: $a-$ изоповерхность $\rho / \rho_{0}=1.1$; $b-$ изолинии $\rho / \rho_{0}$ в горизонтальной плоскости симметрии $(y=0)$.

Это равносильно добавлению плоской волны в плоскости изображения. В результате формируется интерферограмма в бесконечной полосе, что позволяет получать данные о плотности потока. Для интерферограмм в бесконечной полосе $\Delta N_{0}=0$ в выражении (1). На рис. 1, $a$ представлена типичная интерферограмма, полученная в ходе экспериментов.

Трехмерное численное моделирование исследуемого течения выполнено с помощью разработанного в ИТПМ комплекса программ CFS3D (Compressible Flow Solver, Three-Dimensional) для решения уравнений

Письма в ЖТФ, 2017, том 43, вып. 10 
Эйлера и Навье-Стокса, надежно апробированного при исследовании пространственной структуры регулярного и маховского отражения между двумя клиньями или пластинами конечного размаха [7-9]. Численный алгоритм основан на конечно-объемной TVD (Total Variation Diminishing) схеме 4-го порядка. Так же как и в работах [7,8], в расчетах моделировалось течение между двумя симметричными бесконечно тонкими пластинами на основе численного решения трехмерных нестационарных уравнений Эйлера. Исходные данные соответствовали условиям эксперимента $\left(\mathrm{M}=3\right.$, угол отклонения потока $23^{\circ}$, отношение размаха пластины к хорде 3.25). Расчеты проводились методом установления по времени. Критерием установления течения считалась неизменность положения маховского скачка. В расчетах использовалась трехмерная структурированная сетка с общим числом ячеек $7.5 \mathrm{Mлн.}$

На рис. $1, b$ представлена численная интерферограмма, полученная из расчетных данных распределения плотности для соответствующей нашим экспериментам длины волны зондирующего излучения $\lambda=532 \mathrm{~nm}$. Видно, что качественно картина течения хорошо соотносится с результатами, полученными в процессе эксперимента (рис. 1, $a$ ).

При численном моделировании рассчитывалось распределение плотности в безразмерном виде $\rho(x, y, z) / \rho_{0}$, где $\rho_{0}-$ плотность в невозмущенном потоке. Результаты расчетов подтвердили основные особенности трехмерной структуры течения, упомянутые еще в работах $[7,8]$, такие как немонотонность изменения высоты маховского скачка по размаху и существование периферийного маховского отражения (рис. 2). Были выявлены следующие особенности распределения плотности в „виртуальном сопле“ за ножкой Маха: плотность по длине сопла уменьшается, а интегральная по поперечной координате плотность изменяется немонотонно. Несмотря на то что плотность падает, интеграл непосредственно за скачком растет вследствие увеличения размеров пути интегрирования (рис. $2, b$ ).

Сравнение результатов численного моделирования и экспериментальных данных проводилось вдоль оси симметрии течения $(y=0)$ для величины

$$
\Delta L(x)=\int_{0}^{L}\left[\frac{\rho(x, z)}{\rho_{0}}-\frac{\rho\left(x_{b}, z\right)}{\rho_{0}}\right] d z .
$$

Письма в ЖТФ, 2017, том 43, вып. 10 

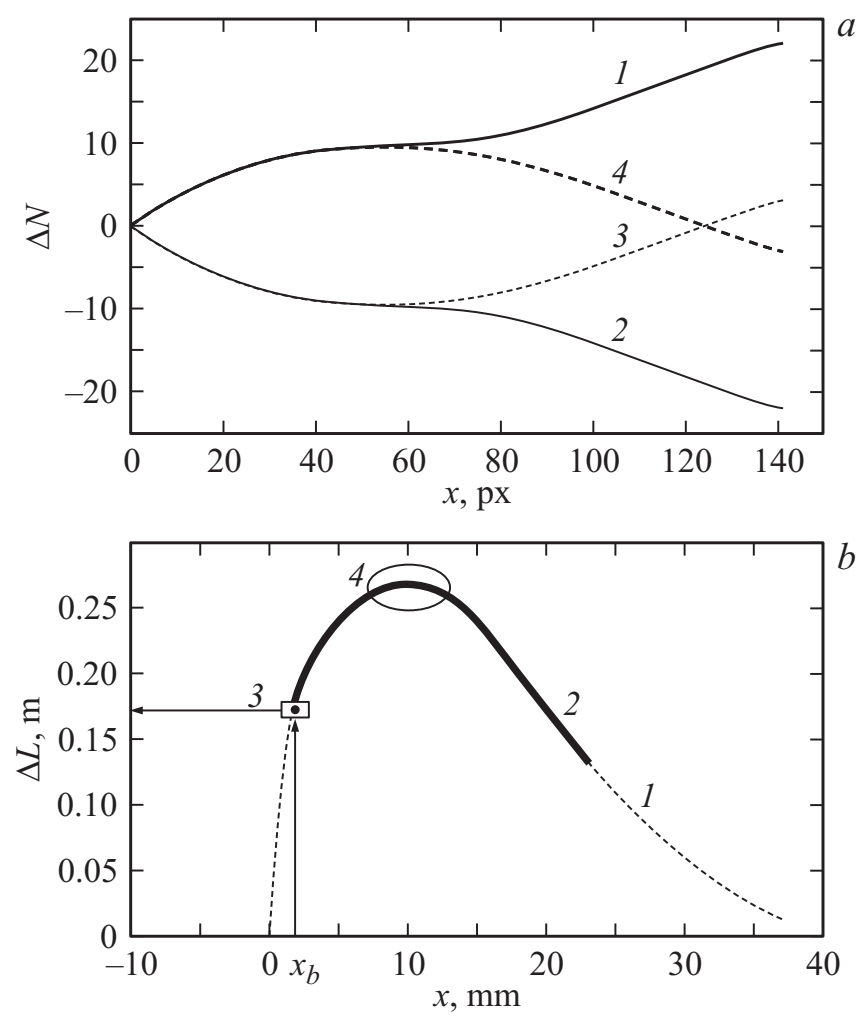

Рис. 3. Сравнение результатов расчета с результатами, полученными по экспериментальным интерферометрическим данным: $a-$ возможные варианты поведения зависимости номера полосы от координаты на изображении (в пикселях); $b-$ распределение интегральной плотности вдоль оси „виртуального сопла“.

Для определения значения $\Delta L(x, y)$ из экспериментальных данных для каждого сечения $x=$ const определялся номер интерференционной полосы относительно базовой точки $x_{b}$, которая соответствовала на интерферограмме первой различимой за ножкой Маха полосе:

$$
\Delta N(x)=N(x)-N\left(x_{b}\right)
$$

Письма в ЖТФ, 2017, том 43, вып. 10 
Далее определялось экспериментальное значение $\Delta N(x)$ по формуле

$$
\Delta L(x)=\frac{\lambda \Delta N(x)}{\xi \rho_{0}} .
$$

Результаты численного моделирования были использованы при трактовке экспериментальных данных. При расшифровке интерферограмм не всегда возможно однозначно определить зависимость номера полосы от координаты: рост, убывание, наличие экстремума. Для выбора этой зависимости необходимы данные о поведении оптического пути, которые не могут быть получены непосредственно из интерферограмм. В нашем случае существует четыре возможных варианта поведения зависимости интегральной плотности (номера интерференционной полосы) от координаты вдоль оси виртуального сопла (рис. 3,a). Эта информация, а также значение $\Delta L\left(x_{b}\right)$ (метка 3 на рис. $3, b$ ) были получены именно из результатов численного моделирования.

На рис. $3, b$ представлено сравнение результатов расчета и расшифровки интерферограмм. Хорошее согласие экспериментальных и расчетных данных подтверждает работоспособность и достоверность результатов, полученных с использованием метода НП АВТ, а также его перспективность. Использование такого комплексного подхода позволило впервые в эксперименте получить количественную информацию о распределении интегральной (по длине оптического пути) плотности и получить необходимые данные для валидации результатов численного моделирования и экспериментальных измерений интегральной плотности.

Работа выполнена при частичной финансовой поддержке РФФИ (№ 16-01-00764).

\section{Список литературы}

[1] Бажсенова Т.В., Гвоздева Л.Г. Нестационарные взаимодействия ударных волн. М.: Наука, 1977. $274 \mathrm{c}$.

[2] Ben-Dor G. Shock Wave Reflection Phenomena. New York: Springer, 2007. $342 \mathrm{p}$.

[3] Гвоздева Л.Г., Гавренков С.А. // Письма в ЖТФ. 2012. Т. 38. В. 8. С. 39-45.

[4] Гавренков С.А., Гвоздева Л.Г. // Письма в ЖТФ. 2012. Т. 38. В. 12. С. 74-80.

[5] Азарова О.А., Гвоздева Л.Г. // Письма в ЖТФ. 2016. Т. 42. В. 15. С. 59-66.

Письма в ЖТФ, 2017, том 43, вып. 10 
[6] Lau-Chapdelaine S.S.-M., Radulescu M. I. // Shock Waves. 2016. V. 26. N 5. P. 551-560.

[7] Ivanov M.S., Vandromme D., Fomin V.M. et al. // Shock Waves. 2001. V. 11. N 3. P. 199-207.

[8] Иванов М.С., Кудрявцев А.Н., Никифоров С.Б., Хотяновский Д.В. // Аэромеханика и газовая динамика. 2002. № 3. С. 3-12.

[9] Ivanov M.S., Kudryavtsev A.N., Nikiforov S.B. et al. // Phys. Fluids. 2003. V. 15. N 6. P. $1807-1810$

[10] Бойко В.М., Оришич А.М., Павлов А.А., Пикалов В.В. Методы оптической диагностики в аэрофизическом эксперименте: Новосибирск: НГУ, 2009. $450 \mathrm{c}$.

[11] Павлов А.А., Голубев М.П., Павлов Ал.А. // Вестн. НГУ. Сер.: Физика. 2014. T. 9. № 1. C. 15-28.

4 Письма в ЖТФ, 2017, том 43, вып. 10 